\title{
SUGLI ERRORI PROGRESSIVI DELLE LETTURE DI UN GRAVIMETRO
}

\author{
Armando Norinelli
}

L'Istituto di Geodesia e Geofisica dell'Università di Padova ha in uso, quale cortese prestito della Societa Montecatini, il gravimetro Western G.4 A, N. 11 di matricola. Tale gravimetro servì a terminare un rilievo gravimetrico dettagliato dell'area eruttiva euganeo-bericolessinea e serve attualmente a completare quello a larghe maglie (1 stazione ogni $100 \mathrm{~km}^{2}$ ) della regione assegnata per tale scopo all'Istituto dalla Commissione Geodetica Italiana.

Poiché la Casa costruttrice del gravimetro ha fornito per esso due costanti di scala: - 0,08515 per le dieci rivoluzioni più hasse del tamburo e -0,08575 per le rimanenti, mi accinsi, con l'approvazione del prof. Silva, ad esaminare più a fondo la variazione della costante di scala determinando gli errori progressivi delle letture. Non ci si riferisce qui agli errori progressivi propri della vite, perché assieme alla non costanza del passo di essa può associarsi fra l'altro una non rigogosa proporzionaliti tra l'allungamento della molla di misura e la variazione della sua reazione elastica, il che conduce ad una non rigrorosa proporzionalita tra le variazioni di lettura al tamburo, the sono proporzionali all'allungamento, e le variazioni di gravità, alle quali è proporzionale la variazione della reazione elastica al momento della lettura. In tale ipotesi il valore della costante di scala dovreble variare in modo uniforme con le letture.

Ciò equivale ad ammettere che tra la differenza di gravita $g-g_{0}$ e di letture al gravimetro $l-l_{0}$ esista una relazione del tipo:

$$
g-g_{0}=\bar{k}\left(l-l_{0}\right)+h\left(l-l_{0}\right)^{2}
$$

con $\vec{k}$ ed $\bar{h}$ costanti. $\bar{h}$ essendo una quantità molto piccola rispetto a $\bar{h}$, $\operatorname{con} l_{0}$ lettura minima possibile al tamburo, con $g_{0}$ valore della gravita ad essa corrispondente, con $l$ lettura corrispondente alla gravità g.

Mantenendo la stessa ipotesi, si pui adottare un'unica costante h: di scala, per avere la stessa differenza di gravita dalla stessa diffe- 
senza di letture, applicando alla lettura variabile $l$ una correzione $\omega$ e lasciando invariata la lettura $l_{0}$. Ciio equivale a scrivere:

$$
g-g_{0}=k\left(l+\omega-l_{\omega}\right) \text {. }
$$

Dalla [1] e [2] per confronto si ricava:

$$
k\left(l-l_{0}\right)+\bar{h}\left(l-l_{0}\right) *=k\left(l+(1)-l_{0}\right) \text {. }
$$

Considerando nulla la correzione anche per la lettura massima possibile $I_{m}$, la [3] diviene:

$$
\bar{k}\left(l_{\mathrm{m}}-l_{\mathrm{o}}\right)+\bar{h}\left(l_{\mathrm{m}}-l_{\mathrm{o}}\right)^{*}=h\left(l_{\mathrm{m}}-l_{\mathrm{o}}\right),
$$

da cui :

$$
h=\bar{h}+\bar{h}\left(l_{\mathrm{m}}-l_{\mathrm{o}}\right)
$$

Tenendo conto di questa relazione, dalla [3] si trae:

$$
(1)=\frac{\bar{h}}{h}\left(l-l_{0}\right)\left(l-l_{\mathrm{m}}\right) \text {. }
$$

Se si rappresenta con un grafico la [4], si ottiene una parabola le cui ordinate stanno ad indicare le correzioni da apportare alle letture rappresentate in ascissa, dovute all'ipotesi di partenza che dipende soltanto dalla non perfetta proporzionalità fra letture ed azione della molla di misura.

Tenendo anche conto della non costanza del passo della vite e di eventuali altre cause e adottando la formula elementare $g-g_{0}=k$ $l l-l_{0}$, si avrà in corrispondenza ad ogni lettura $l$ un errore $\varepsilon$ che potremo chiamare errore progressivo, dato che tale denominazione usuale nelle analoghe ricerche sulla regolarita del passo di una vite. Tale errore è uguale e contrario alla correzione $-\varepsilon$, da applicare ad $l$ per avere la lettura ideale che si farebhe con uno strumento perfetto.

Al fine di ottenere tali errori per il gravimetro Western, tipo G 4 A, N. 11 di matricola, si è seguito il procedimento seguente.

Precisiamo anzitutto che il tamburo è stato fissato alla vite corrispondente per modo che, quando il sistema tamburo-vite è completamente avvitato, la lettura massima è 1527 , e poiché la lettura minima utilizzabile è di poco inferiore a 300 , venne limitata la ricerca all'intervallo definito dalle letture estreme $320-1520$. Tale intervallo fu suddiviso in 6 sezioni, corrispondenti ciascuna a quattro rivoluzioni del tamburo, separate fra loro dalle letture: $1320,1120,920,720,520$. 
Vennero quindi scelte sulla strada che sale al Monte Venda (Padova) 7 stazioni in modo che la differenza di gravità tra due stazioni consecutive fosse approssimativamente la stessa e tale da imporre al tamburo per la misura quattro rivoluzioni nello stesso senso. Le osservazioni vennero disposte secondo lo schema che segue, nel quale i numeri romani stanno ad indicare le successive stazioni ed i numeri arabici, posti vicino alla prima e allultima stazione di ogni serie, indicano le letture convenzionali prossime a quelle effettivamente avute in dette stazioni. La lettura fatta nella prima stazione venne di volta in volta imposta al tamburo, agendo sulla vite che serve a variare il campo di misura (reset); la differenza tra la lettura prefissata e quella effettivamente ottenuta rimase sempre entro i limiti di due-cinque divisioni del tamburo.

I (1320), II (1520), II, I

I $(1120)$, II, III $(1520)$, III, II, I

I 1920$)$, II, III, IV $(1520)$, IV, III, II, I

I $(720)$, II, III, IV, V $(1520), \mathrm{V}$, IV, III, II, I

I $(520), \mathrm{II}, \mathrm{III}, \mathrm{IV}, \mathrm{V}, \mathrm{VI}(1520), \mathrm{VI}, \mathrm{V}, \mathrm{IV}, \mathrm{III}, \mathrm{II}, \mathrm{I}$

I (320), II, III, IV, V, VI, VII (1520), VII, VI, V, IV, III, II, I

II (320) III, IV, V, VI, VII (1320). VII. VI, V, IV, III, II

III (320), IV, V, VI, VII (1120), VII, VI, V, IV, III

IV $(320), \mathrm{V}, \mathrm{VI}$, VII $(920)$, VII, VI, V, IV

V $(320)$, VI, VII $(720)$, VII, VI, V

VI $(320)$, VII $(520)$, VII, VI.

In questo modo ciascuna delle sei differenze di gravità tra due stazioni successive venne misurata due volte (in andata e in ritorno) in corrispondenza di ciascuna delle sei sezioni della vite.

Qualora le letture non fossero affette da errori progressivi, indicata con $\triangle g_{\mathrm{i}}(i=1,2,3.4,5,6)$ la differenza di gravità tra le due stazioni $i^{\text {exima }}$ e $(\imath+1)^{\text {mima }}$, con $l_{\mathrm{h}, i}, l_{\mathrm{h}-1, \mathrm{i}-1}(h=1,2,3,4,5,6)$ la coppia di letture con eui renne misurata la differenza di gravita $\Delta g_{1}$ in corrispondenza della sezione $h^{\text {esimil }}$ della vite $\left(l_{1} \sim 320, \ldots \ldots, l_{\gamma} \sim 1520\right.$ ) e con $k$ la costante del gravimetro, dovrebbe essere, a meno degli errori di osservazione:

$$
\left(I_{\mathrm{h}+\mathrm{i} \cdot \mathrm{i}+\mathrm{i}}-I_{\mathrm{h} \cdot \mathrm{i}}\right) k=\Delta g_{\mathrm{i}}
$$

In causa degli errori progressivi $\Sigma_{11}(h=1,2,3,4,5,6)$, tale equazione risulterà invece soddisfatta, sempre a meno degli errori di osserva- 
zione, qualora alle letture $l_{\mathrm{h}}$ venqano apportate le corrispondenti correzioni $-\varepsilon_{\mathrm{h}}$, sicché si dovra serivere:

$$
\left(l_{\mathrm{h}+1 \cdot+\mathrm{i}+1}-\varepsilon_{\mathrm{l}+\mathrm{i}+1}\right)-\left(l_{1 \cdot \mathrm{i}}-\varepsilon_{\mathrm{h}}\right)=\frac{\Delta g_{\mathrm{i}}}{k} .
$$

Assumendo per $k$ il valore medio della costante del gravinetro per tutta l'escursione da 320 a 1520 della vite, gli errori progressivi $\varepsilon_{1}$ ed $\varepsilon_{\text {- }}$ corrispondenti a tali letture estreme dovranno essere equali e possiamo ritenerli nulli.

Se allora si rimiscono in media le 6 equazioni corrispondenti ad un medesimo indice $i$, variando l'indice $h$, e si sottrae nembro a menbro dalla precedente l'equazione che ne risulta, si ottiene:

$$
\varepsilon_{\mathrm{h}+1}-\varepsilon_{\mathrm{h}}=\left(l_{\mathrm{h}+1 \cdot \mathrm{i}-\mathrm{-}}-l_{\mathrm{h} \cdot \mathrm{i}}\right)-\frac{1}{6} \stackrel{!}{\mathrm{l}}_{\mathrm{h}}\left(l_{\mathrm{h}-1 \cdot \mathrm{i}-\mathrm{l}}-l_{\mathrm{h} \cdot \mathrm{i}}\right) .
$$

Lo specchio che riporta le differenze $l_{\mathrm{h}-1 \cdot \mathrm{i}-1}-l_{\mathrm{h} \cdot \mathrm{i}}, \Sigma_{\mathrm{h}-1}-\Sigma_{\mathrm{h}}$,

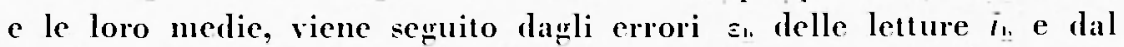
grafico a linea continua (fig. 1) delle correzioni $-\varepsilon_{11}$ a tali letture.

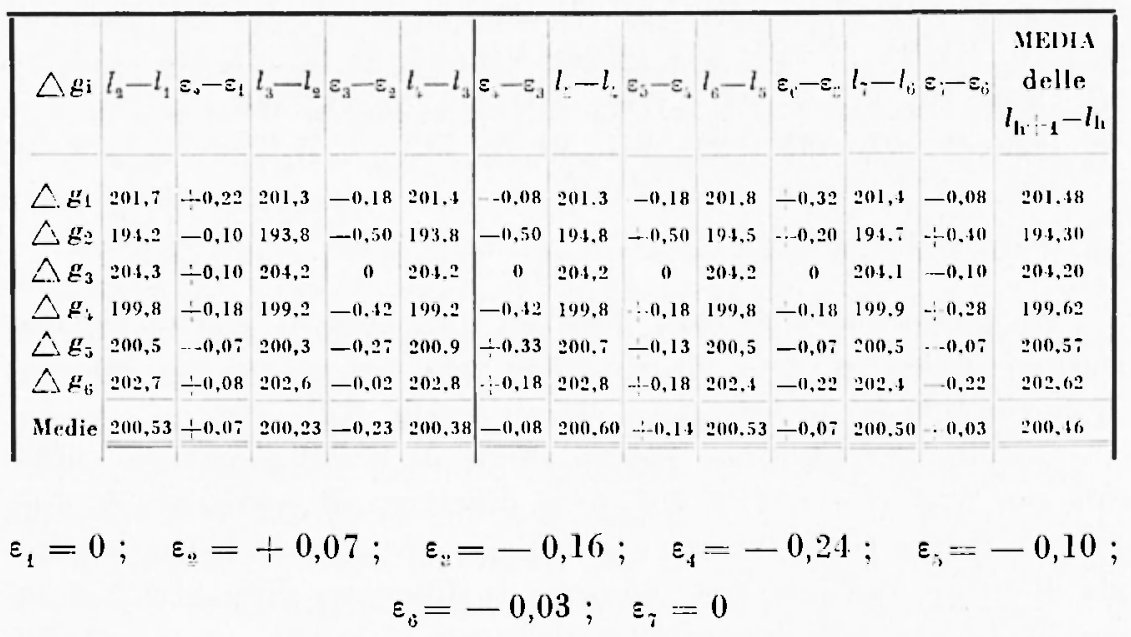

Indicando con $v$ grli scostamenti delle differenze $\varepsilon_{\mathrm{h}-,}-\varepsilon_{\mathrm{h}}$ dalla loro media, il quadrato dell'error medio y di una differenza $i$ dato da:

$$
v^{2}=\frac{\lceil v v\rceil}{n-m}=0,053
$$

dove $n=36$ (numero dei $v$ । e $m=6$ (numero delle medie). 
Indicando con $\mu$ il quadrato dell'error medio di una differenza $l_{\mathrm{h}+1 \cdot \mathrm{i}-\}_{-}}-l_{\mathrm{h} \cdot \mathbf{i}}$ dello specchio surriportato, si ricava con facili passaggi, applicando alla [5] la formula di propagazione degli errori accidentali :

$$
\mu^{2}=\frac{6}{5} v^{2}=0,063
$$

Ed essendo $l_{\mathrm{h}+1 \cdot \mathrm{i}+1}-l_{\mathrm{h} \cdot \mathrm{i}}$ media di due valori, l'errore medio di una differenza $l_{\mathrm{h}+1 \cdot \mathrm{i}+1}-l_{\mathrm{h} \cdot \mathrm{i}}$ misurata una volta sola risulta $+0,35 \mathrm{e}$ l'error medio di un lettura $+0,25$. Poiché si fecero sempre 3 letture al gravimetro, tale errore medio è da attribuirsi in maniera prepon-

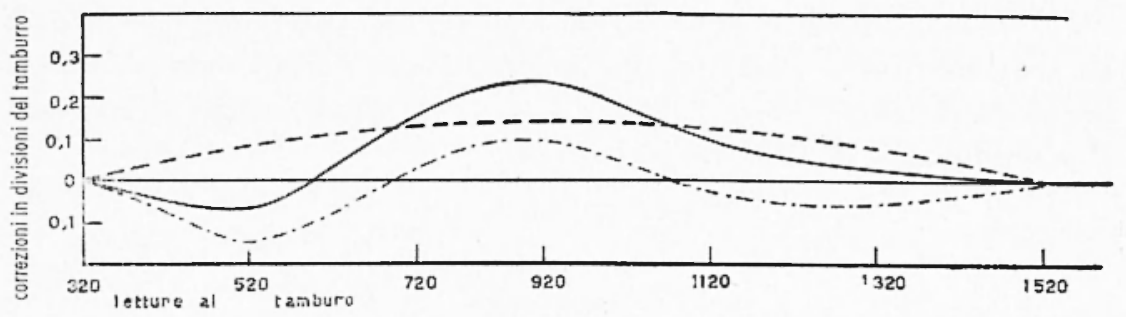

Fig. 1

derante alla rispondenza dello strumento alle misure nella suesposta ricerca ed è quindi da riguardarsi piuttosto come l'errore medio " proprio " del Western N. 11, quando è usato in buone condizioni di lavoro. Al detto error medio $\pm 0,25$ della lettura, corrisponde un'incertezza di $\therefore 0,02 \mathrm{mgal}$ in una osservazione di gravità. Dato che gli errori progressivi non superano l'errore medio di lettura, non è certo il caso di tener conto di essi ed è altresì da escludere l'esistenza dei due valori distinti lati dalla Casa costruttrice per la costante di scala del gravimetro.

A titolo di curiosità si è voluto applicare a questo caso le considerazioni svolte all'inizio. Nell"ipotesi in cui fosse applicabile la [4], dove $l_{\mathrm{o}}=320, l_{\mathrm{m}}=1520$ ed $\omega=-\varepsilon_{\mathrm{h}}$, risolvendo col metodo delle osservazioni mediate il sistema che si ottiene sostituendo alla $\omega$ successivamente le correzioni calcolate $-\varepsilon_{\mathrm{h}}$ ed alla $l$ le letture corrispondenti $l_{1}$, si deduce l'equazione:

$$
\omega=-0,39 \cdot 10^{-6}(l-320)(l-1520) \text {, }
$$


che nella fig. 1 è rappresentata dall'arco di parabola tratteggiato. Dal confronto dei due grafici si deve escludere che gli errori siano dovuti esclusivamente al difetto della molla di misura considerato; si riconosce tuttavia che accettando la detta ipotesi e togliendo dacrli errori progressivi osservati quelli dovuti alla molla, grli errori residui sono notevolmente attenuati, come appare nella curva a tratio e punto della fig. 1 .

Padova - Istituto di Geodesia e Geofisica - Maggio 1954.

\section{RIASSUNTO}

Viene precisato il concetto di errori progressivi delle letture di un gravimetro e si determina sperimenta!mente lammontare di questi per il gravimetro Western, tipo G $4 . \mathrm{A}, \mathrm{N} .11$ di matricola, esponendo il procedimento di lavoro e di calcolo.

\section{SUMMARY}

The concept of progressive errors in the reading of a gravimeter is defined and the sum of these errors is calculated for the Western gravimeter, type $G 4 A$, Cat. No. 11. The working procedure is set forth in detail. 